\title{
Effect of the Biofilm Age and Starvation on Acid Tolerance of Biofilm Formed by Streptococcus mutans Isolated from Caries-Active and Caries-Free Adults
}

\author{
Shan Jiang ${ }^{1,+}$, Shuai Chen ${ }^{1,+}$, Chengfei Zhang ${ }^{2}$, Xingfu Zhao ${ }^{3}$, Xiaojing Huang ${ }^{1, *}$ and Zhiyu Cai ${ }^{4}$ \\ 1 Department of Endodontics and Operative Dentistry, School and Hospital of Stomatology, \\ Fujian Medical University, 246 Yangqiao Zhong Road, Fuzhou 350002, China; kqshanj@163.com (S.J.); \\ drchenshuai@163.com (S.C.) \\ 2 Department of Endodontics, Comprehensive Dental Care, Faculty of Dentistry, \\ The University of Hong Kong, Hong Kong, China; zhangcf@hku.hk \\ 3 Department of Endodontology, Tianjin Stomatological Hospital of Nankai University, 75 North Dagu Road, \\ Heping District, Tianjin 300000, China; xingfu_108@126.com \\ 4 Department of Oral and Maxillofacial Surgery, Fujian Medical University Union Hospital, \\ Fuzhou 350001, China; caizhiyu2007@126.com \\ * Correspondence: hxiaoj@163.com; Tel.: +86-591-8373-6419 \\ + These authors contributed equally to this work.
}

Academic Editor: Martin Welch

Received: 18 February 2017; Accepted: 22 March 2017; Published: 30 March 2017

\begin{abstract}
Streptococcus mutans (S. mutans) is considered a leading cause of dental caries. The capability of $S$. mutans to tolerate low $\mathrm{pH}$ is essential for its cariogenicity. Aciduricity of $S$. mutans is linked to its adaptation to environmental stress in oral cavity. This study aimed to investigate the effect of biofilm age and starvation condition on acid tolerance of biofilm formed by S. mutans clinical isolates. S. mutans clinical strains isolated from caries-active (SM593) and caries-free (SM18) adults and a reference strain (ATCC25175) were used for biofilm formation. (1) Both young and mature biofilms were formed and then exposed to $\mathrm{pH} 3.0$ for $30 \mathrm{~min}$ with (acid-adapted group) or without (non-adapted group) pre-exposure to $\mathrm{pH} 5.5$ for three hours. (2) The mature biofilms were cultured with phosphate-buffered saline (PBS) (starved group) or TPY (polypeptone-yeast extract) medium (non-starved group) at $\mathrm{pH} 7.0$ for $24 \mathrm{~h}$ and then immersed in medium of $\mathrm{pH} 3.0$ for $30 \mathrm{~min}$. Biofilms were analyzed through viability staining and confocal laser scanning microscopy. In all three strains, mature, acid-adapted and starved biofilms showed significantly less destructive structure and more viable bacteria after acid shock than young, non-adapted and non-starved biofilms, respectively (all $p<0.05$ ). Furthermore, in each condition, SM593 biofilm was denser, with a significantly larger number of viable bacteria than that of SM18 and ATCC25175 (all $p<0.05$ ). Findings demonstrated that mature, acid-adapted and starvation might protect biofilms of all three $S$. mutans strains against acid shock. Additionally, SM593 exhibited greater aciduricity compared to SM18 and ATCC25175, which indicated that the colonization of high cariogenicity of clinical strains may lead to high caries risk in individuals.
\end{abstract}

Keywords: Streptococcus mutans; biofilm formation; aciduricity; Starvation; confocal laser scanning microscopy

\section{Introduction}

Dental caries is an infectious and transmissible bacterial disease [1,2]. Streptococcus mutans (S. mutans) has been described as the principal etiologic agent of human dental caries and an important constituent of dental plaque [3]. S. mutans varies in cariogenicity as a result of different growing 
conditions such as carbohydrate source, $\mathrm{pH}$, biofilm presence, starvation, and slight variations in different species [4]. It is widely accepted that strong capability of S. mutans to tolerate low $\mathrm{pH}$, namely aciduricity, is essential for its cariogenicity [5]. The constitutive acid tolerance properties of S. mutans, as well as its adaptive acid tolerance response (ATR) provide a holistic benefit to withstand continual changes in acid shock [6]. It has been reported that ATR plays a crucial role in protecting S. mutans from more severe acid stress [7]. In other words, acid adaption triggered by prior exposure to low but nonlethal $\mathrm{pH}$ enables $S$. mutans to survive in severe acidification.

Moreover, biofilm has significant effects on the aciduricity of the bacteria because of the complexity of the oral environment [8]. Several studies have shown that S. mutans in biofilms have increased adaptive capabilities to adverse conditions and display increased tolerance to antimicrobial treatments $[9,10]$. For example, biofilm cells were tremendously more tolerant to acid stress, with a $41.5 \%$ survival rate, while such rate was only $0.00004 \%$ for planktonic cells [11]. Interestingly, starvation also has been suggested to induce the tolerance of planktonic bacteria to acid shock [12]. Thus, bacteria in oral biofilm have been speculated to be more tolerant to acid during the starvation between meals [13].

On the other hand, it is worth mentioning that mutans streptococcus (MS) are found in all peoples regardless of race, ethnic background or geographic origin, with $S$. mutans (serotype c) as majority. Normally, S. mutans is present in the mouth as an insignificantly small component of oral flora. When people have bad oral hygiene and unhealthy diet for a long time, S. mutans may become a dominant member in plaque flora and cause caries [14]. However, the amount of S. mutans is not always parallel with caries level [15]. A growing body of evidences supports that even the same serotype c $S$. mutans clinical isolates could be genetically different $[16,17]$. Additionally, how these differences affect virulence properties of cariogenic $S$. mutans clinical isolates is still poorly understood.

To answer these questions, we previously examined one patient with multiple active carious lesions, and the level of $S$. mutans in his saliva was not high enough to explain his situation. We then acquired a plaque sample from his healthy smooth enamel surface, not from carious lesions, considering that $S$. mutans are most strongly associated with the onset of caries [18-20]. Using polymerase chain reaction (PCR), we identified five S. mutans (serotype c) clinical isolates from this sample. We found that both their genotypes and virulence properties were different. One of the clinical isolates, named SM593, has already been isolated previously from the caries-active adult. Compared with SM18 from the caries-free adult, SM593 grew faster and was more competent in adhesion and biofilm formation [21-24]. However, limited information is now available on the differences in the aciduricity of their biofilms under different conditions.

In the present study, we examined the biofilms of S. mutans clinical strains SM593 and SM18 and the standard strain ATCC 25175 in terms of aciduricity in different conditions including biofilm formation in different phases (young and mature biofilms), acid adaptation (acid-adapted and non-adapted biofilm) and starvation (starved and non-starved biofilms) using confocal laser scanning microscopy (CLSM) with molecular probes LIVE/DEAD BacLight viability stain.

\section{Results}

\subsection{Effect of ATR on Acid Tolerance of S. mutans Biofilm}

To determine the effect of ATR on acid survival of biofilm cells of $S$. mutans, acid adaptation experiment was conducted by exposing biofilms to $\mathrm{pH} 5.5$ for $3 \mathrm{~h}$ and then exposing to $\mathrm{pH} 3.0$ for $30 \mathrm{~min}$ (acid-adapted group), and exposing biofilms to $\mathrm{pH} 3.0$ for $30 \mathrm{~min}$ without prior acid adaptation as comparative controls (non-adapted group). The differences in morphological feature and viability of biofilms were compared and summarized as following. 


\subsubsection{Differences in Acid Tolerance between the Acid-Adapted and Non-Adapted Biofilms}

As shown in Figure 1, both young and mature biofilms of all three strains (ATCC 25175, SM18, and SM593) in the non-adapted group were dominantly composed of dead cells (red color), whereas young and mature biofilms of all three strains in the acid-adapted group were composed mainly of live cells (green), forming distinct network shapes. Additionally, as shown in Tables 1 and 2, the viability in each layer (inner, middle and outer) of young and mature biofilms in the acid-adapted group was significantly higher than that of in the non-adapted group (all $p<0.05$ ). Lastly, the differences between three different layers were compared. In general, the order of the viability among three layers was: middle layer > inner layer > outer layer. Specifically, within each group, the number of viable biofilms cells in the middle layer was significantly more than that of in the outer and inner layers (all $p<0.05)$, and no significant difference was found between inner and outer layers (all $p>0.05$ ).
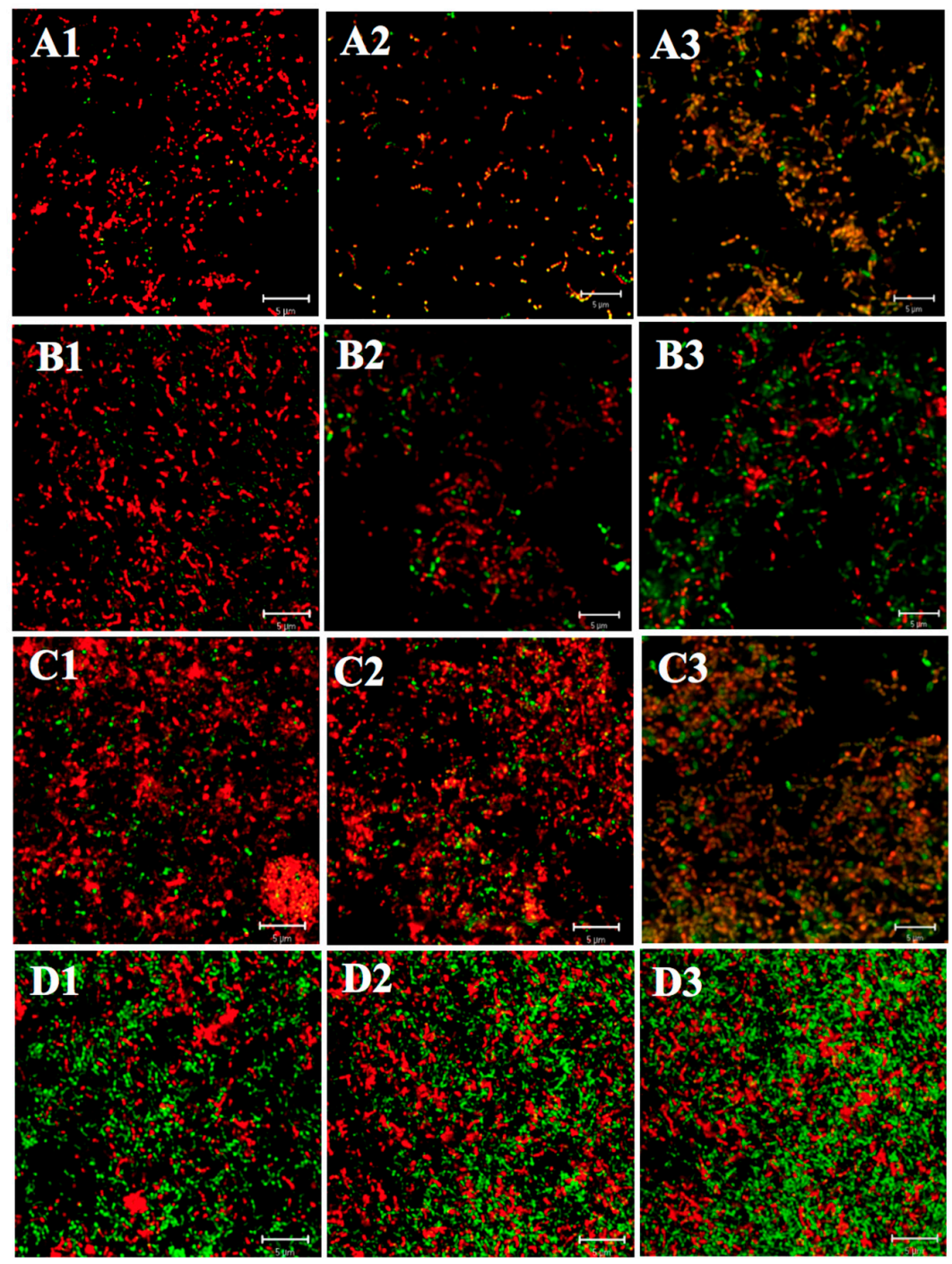

Figure 1. Confocal laser scanning microscopy (CLSM) images of Live/Dead (SYTO-9 and PI) stained young and mature biofilms treatment with acid-adapted or non-adapted. Young biofilm in non-adapted group (A1), ATCC25175; (A2), SM18; (A3), SM593; Young biofilm in acid-adapted group (B1), ATCC25175; (B2), SM18; (B3), SM593; Mature biofilm in non-adapted group (C1), ATCC25175; (C2), SM18; (C3), SM593; Mature biofilm in acid-adapted group (D1), ATCC25175; (D2), SM18; (D3), SM593 (scale bars, 5 mm; magnification $630 \times$ ). 
Table 1. The viability of three strains in different layers of young biofilms (mean $\pm \mathrm{SD}$ ).

\begin{tabular}{ccccccc}
\hline \multirow{2}{*}{ Strains } & \multicolumn{2}{c}{ Outer } & \multicolumn{2}{c}{ Middle } & \multicolumn{2}{c}{ Inner } \\
\cline { 2 - 6 } & Non-Adapted & Acid-Adapted & Non-Adapted & Acid-Adapted & Non-Adapted & Acid-Adapted \\
\hline ATCC25175 & $3.15 \pm 0.67$ & $11.12 \pm 0.85$ & $13.27 \pm 1.17$ & $24.17 \pm 1.01$ & $4.34 \pm 0.91$ & $11.85 \pm 0.75$ \\
SM18 & $2.56 \pm 0.79$ & $10.91 \pm 0.92$ & $12.45 \pm 0.95$ & $21.42 \pm 0.95$ & $3.23 \pm 0.96$ & $10.99 \pm 0.81$ \\
SM593 & $7.72 \pm 0.57$ & $15.41 \pm 0.96$ & $22.56 \pm 0.87$ & $42.37 \pm 0.44$ & $8.51 \pm 0.91$ & $15.65 \pm 0.65$ \\
\hline
\end{tabular}

Table 2. The viability of three strains in different layers of mature biofilms (mean \pm SD).

\begin{tabular}{ccccccc}
\hline \multirow{2}{*}{ Strains } & \multicolumn{2}{c}{ Outer } & \multicolumn{2}{c}{ Middle } & \multicolumn{2}{c}{ Inner } \\
\cline { 2 - 7 } & Non-Adapted & Acid-Adapted & Non-Adapted & Acid-Adapted & Non-Adapted & Acid-Adapted \\
\hline ATCC25175 & $12.26 \pm 0.22$ & $18.43 \pm 1.23$ & $29.72 \pm 0.97$ & $49.88 \pm 0.87$ & $12.49 \pm 0.74$ & $19.76 \pm 0.76$ \\
SM18 & $11.48 \pm 0.75$ & $17.52 \pm 1.11$ & $28.71 \pm 1.04$ & $47.79 \pm 0.83$ & $13.42 \pm 0.85$ & $18.52 \pm 1.16$ \\
SM593 & $16.51 \pm 0.96$ & $31.19 \pm 1.06$ & $38.73 \pm 0.74$ & $68.75 \pm 0.95$ & $17.31 \pm 0.76$ & $34.45 \pm 1.36$ \\
\hline
\end{tabular}

\subsubsection{Differences in Acid Tolerance between Young and Mature Biofilms}

To assess whether the biofilm age influences the aciduricity of biofilm cells of S. mutans, the viability and morphology of young and mature biofilms (formed after $3 \mathrm{~h}$ and $24 \mathrm{~h}$ incubation, respectively) were compared. For all tested strains, in both the acid-adapted and non-adapted groups, structure disruption of young biofilms was greater than that of mature biofilms (Figure 1), and the viability rates for cells in each layer of mature biofilms were significantly higher than those of young biofilms (Tables 1 and 2) (all $p<0.05)$.

\subsubsection{Differences in Acid Tolerance on ATR among Three Strains}

As seen in Figure 1, in either the acid-adapted or non-adapted group, the architecture of both young and mature biofilms formed by SM593 appeared to have relatively less loss of integrity than that of SM18 and ATCC 25175. In addition, as shown in Tables 1 and 2, the viable cells in each layer of young and mature biofilms formed by SM593 were significantly more than those by SM18 and ATCC 25175 (all $p<0.05$ ), while there was no statistical difference between SM18 and ATCC 25175 biofilms (all $p>0.05)$.

\subsection{Effect of Starvation on Acid Tolerance of S. mutans Biofilm}

To examine the effect of starvation on aciduricity of biofilm cells of S. mutans, starvation experiment was performed as described in the Materials and Methods section. Comparison of architecture and viability of starved biofilm (formed by incubating with PBS for $24 \mathrm{~h}$ ) and non-starved biofilm (formed by incubating as normal) exposed to $\mathrm{pH} 3.0$ for 30 min were made.

\subsubsection{Differences in Acid Tolerance between the Starved and Non-Starved Biofilms}

As shown in Figure 2, the biofilm architecture of all three tested strains (ATCC 25175, SM18, and SM593) seemed comparatively less destructive in the starved group than in the non-starved group. Furthermore, the number of viable cell in each layer of starved biofilms was significantly larger than that of non-starved biofilms (Table 3) (all $p<0.05$ ). Also, the comparisons of the viability of three different layers within each group (starved and non-starved groups) showed a similar trend as the ATR on acid tolerance. 

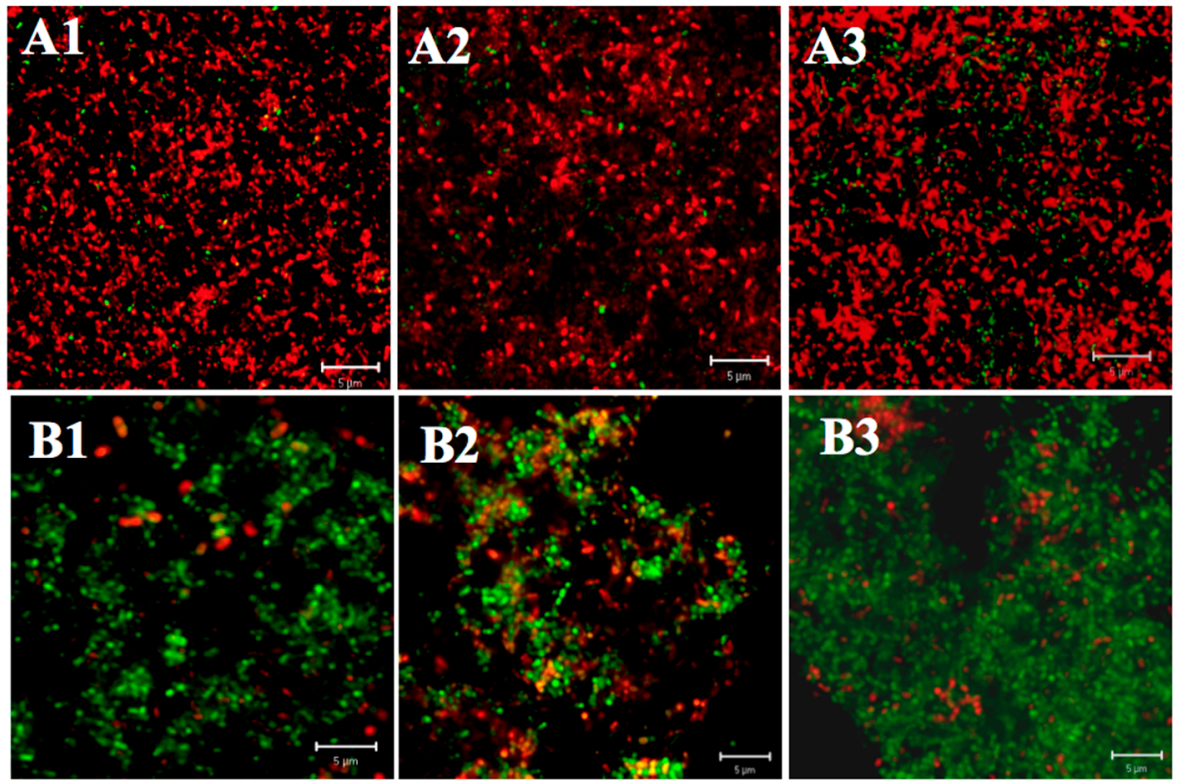

Figure 2. CLSM images of Live/Dead (SYTO-9 and PI) stained starved/non-starved biofilms after acid shock. Non-starved biofilm ((A1), ATCC25175; (A2), SM18; (A3), SM593); Starved biofilm ((B1), ATCC25175; (B2), SM18; (B3), SM593) (scale bars, 5 mm; magnification 630×).

Table 3. The viability of three strains in different layers of starved biofilms (mean $\pm \mathrm{SD}$ ).

\begin{tabular}{ccccccc}
\hline \multirow{2}{*}{ Strains } & \multicolumn{2}{c}{ Outer } & \multicolumn{2}{c}{ Middle } & \multicolumn{2}{c}{ Inner } \\
\cline { 2 - 7 } & Non-Starved & Starved & Non-Starved & Starved & Non-Starved & Starved \\
\hline ATCC25175 & $13.36 \pm 1.05$ & $21.67 \pm 0.66$ & $27.21 \pm 1.05$ & $47.88 \pm 0.87$ & $14.77 \pm 0.76$ & $23.58 \pm 0.88$ \\
SM18 & $11.39 \pm 1.10$ & $22.47 \pm 0.91$ & $26.16 \pm 1.15$ & $47.24 \pm 1.25$ & $12.14 \pm 0.21$ & $23.28 \pm 1.07$ \\
SM593 & $21.47 \pm 1.30$ & $41.59 \pm 0.99$ & $39.28 \pm 0.89$ & $61.31 \pm 0.76$ & $23.11 \pm 0.31$ & $43.35 \pm 0.92$ \\
\hline
\end{tabular}

\subsubsection{Differences in Acid Tolerance on Starvation among Three Strains}

SM593 biofilm had a less likely loss of architectural integrity with significantly higher viability at each layer than S. mutans ATCC 25175 and SM18 in both starved and non-starved groups (all $p<0.05)$, whereas no significant difference was found between ATCC 25175 and SM18 biofilms (all $p>0.05)$ (Figure 2, Table 3).

\subsection{Three-Dimensional Reconstructions}

The three-dimensional image reconstruction of each biofilm was obtained through the series CLSM images. As shown in Figure 3, for all three tested strains, the structures of acid-adapted (both mature and young biofilms) and starved biofilms were relatively flatter and thicker compared to gully shape structure of non-adapted and non-starved ones. Furthermore, in all cases, SM593 biofilm was less likely destructive and denser than that of ATCC 25175 and SM18. 

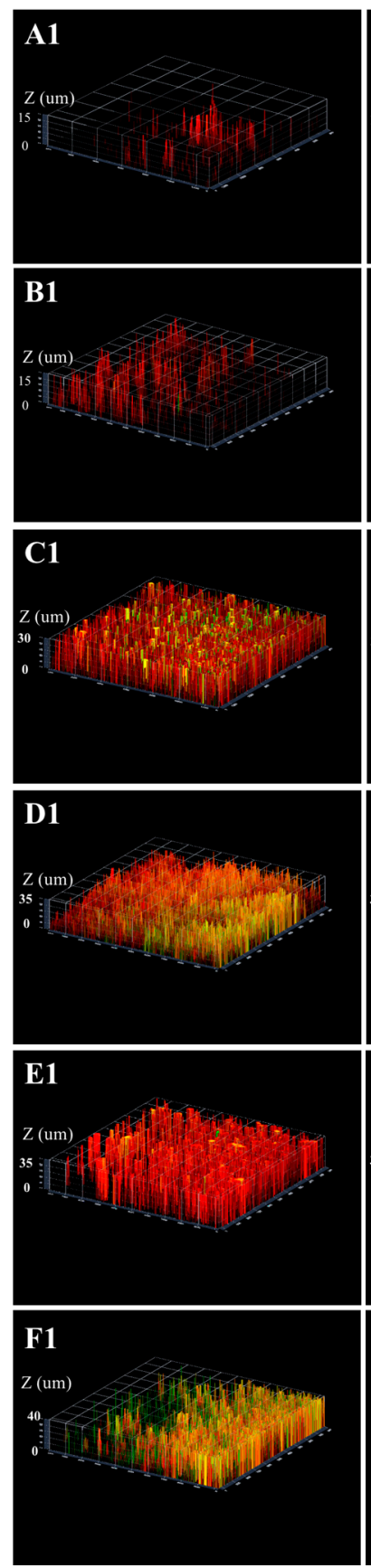
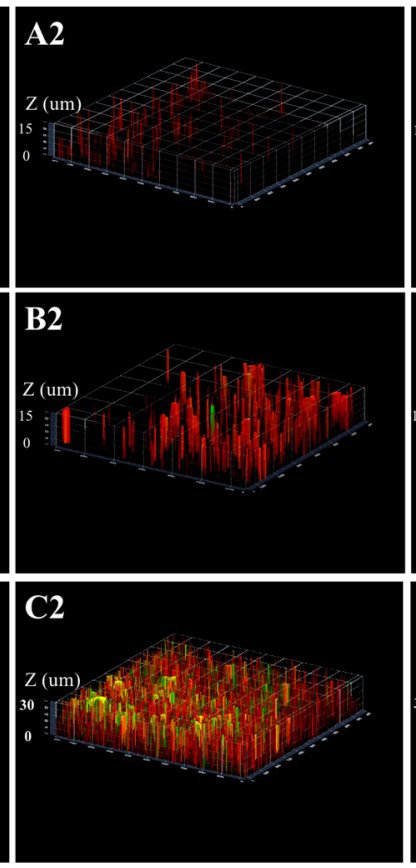

D2
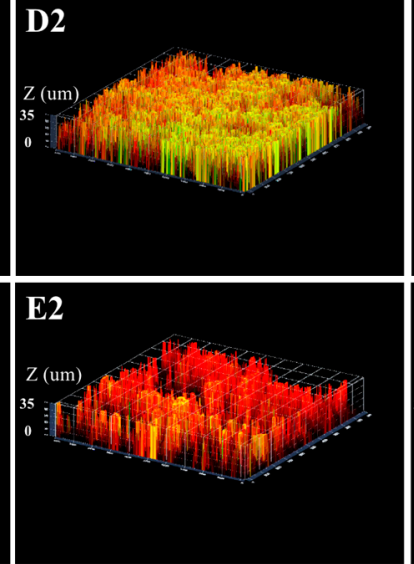

F2

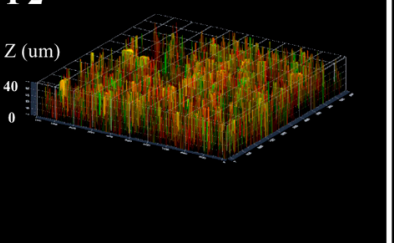

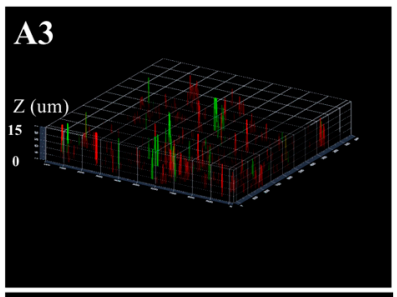

B3
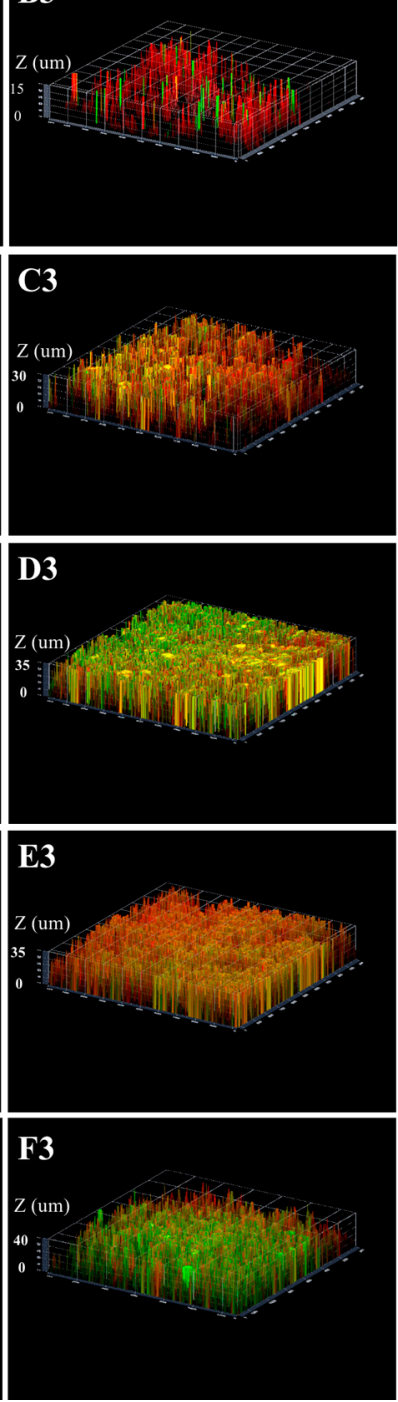

Figure 3. Three-dimensional architecture of $S$. mutans biofilms under different conditions. Young biofilm in non-adapted group (A1), ATCC25175; (A2), SM18; (A3), SM593; Young biofilm in acid-adapted group (B1), ATCC25175; (B2), SM18; (B3), SM593; Mature biofilm in non-adapted group (C1), ATCC25175; (C2), SM18; (C3), SM593; Mature biofilm in acid-adapted group (D1), ATCC25175; (D2), SM18; (D3), SM593 Non-starved biofilm (E1), ATCC25175; (E2), SM18; (E3), SM593; Starved biofilm (F1), ATCC25175; (F2), SM18; (F3), SM593.

\section{Discussion}

S. mutans is generally considered to be a principal etiological agent of dental caries. Variables of S. mutans have been investigated for their associations with the caries process. The ability of $S$. mutans to survive and grow in low $\mathrm{pH}$ environments is fundamental for its survival and eventual dominance in dental plaque, finally leading to caries. In other words, aciduricity of $S$. mutans plays a crucial 
role in the occurrence and development of dental caries and acts as an important virulence factor. Nevertheless, understanding on determination of aciduric virulence factor of $S$. mutans, especially for S. mutans in biofilm lifestyle, is limited. In the present study, S. mutans clinical strains isolated from caries-active (SM593) and caries-free (SM18) adults and the standard strain ATCC 25175 have been used to analyze the association between the aciduricity of strains and caries activity under different conditions (including biofilm age, ATR and starvation) to identify potential factors influencing the aciduricity of $S$. mutans.

The capacity of $S$. mutans to initiate caries via acid production from the metabolism of dietary carbohydrates [18] would be suicidal if not for its remarkable ability to tolerate acid, signifying a crucial aspect of its virulence [25]. In order to withstand these continual cycles of acid shock, S. mutans has evolved a repertoire of mechanisms that fall under two distinct categories; constitutive mechanisms and acid-induced mechanisms [11], also referred to as acid tolerance response (ATR) [7]. More specifically, the ATR of this microorganism is defined as the ability to adapt to acid stress by prior exposure to a low, sub-lethal $\mathrm{pH}$ of approximately 5.5 [25-27]. In the present study, based on the qualitative and quantitative analysis of CLSM data, we found that ATR could remarkably enhance the aciduricity of biofilms generated by biofilms SM593, SM18, and S. mutans ATCC 25175. This finding is consistent with those from previous studies demonstrating that the aciduricity of $S$. mutans in planktonic status could be enhanced by ATR and confirming the ability of $S$. mutans to induce ATR that enhanced its survival at a low $\mathrm{pH}[6,11,26]$. Pre-acidification of sub-lethal $\mathrm{pH} 5.5$ exposure for three hours prior to acid killing induced a stimulon which enhanced S. mutans survival at $\mathrm{pH} 3.0$ [25-27]. Furthermore, the rapid acidification is also followed by the expression of numerous proteins, including chaperones and membrane proteins, all of which were necessary for cellular viability $[28,29]$. However, these possible mechanisms need to be confirmed by further study.

In addition, one of essential factors for viability is the regulation of formed biofilm. Previous studies investigated the effect of chlorhexidine gluconate on treating biofilms reported that the proportion of killed bacteria was much higher in young biofilms than in mature biofilms [30,31]. Likewise, results of the present study demonstrated that whether acid-adapted or non-adapted, the decrease in survivors and structure disruption were more significant in young biofilms than in the matures. It was indicated that mature biofilms might be more likely to prevent biofilm disruption and to offer stronger protection for cells under stress environments (such as acid killing), which were in keeping with findings from earlier works reporting that biofilm cells were more tolerant to environmental stress and antimicrobial agents than their young counterparts [9,32]. One of the possible explanations for this result is that mature biofilm is denser, more compact and complex compared with young biofilm, so it is harder for acid to penetrate. The finding supports that mature biofilms are more cariogenic than young ones. Therefore, besides the well-known fact that mature biofilm is more difficult to treat than young biofilm, removing dental plaque biofilm via cleaning techniques, such as regular toothbrushing and the use of dental flossing in early stage, is highly recommended.

Reportedly, starvation provided cells of Escherichia coli with an equivalent or even slightly higher level of acid tolerance than log-phase ATR [12]. Zhu et al. found that starvation could successfully induce the tolerance of planktonic S. mutans to acid shock at $\mathrm{pH} 3.8$ [13]. Similarly, the results from the present study indicated that starvation enhanced acid tolerance of $S$. mutans in biofilm regardless of the genotypes of strains, supporting the speculation that oral bacteria in dental biofilm plaque is likely to be more tolerant to acid stress during starvation between meals [4]. This could partly explain the phenomenon in daily life that eating sugar or food high in sugar between meals increases the risk of dental caries [33].

On the other hand, in the presence or absence of adaptation and starvation, comparisons for any stage of biofilm between three tested strains of S. mutans indicated that SM593 biofilm was more acid-tolerant and induced a stronger ATR than SM18 and ATCC 25175 biofilms. This result is consistent with the finding that different strains of $S$. mutans exhibited different degrees of low $\mathrm{pH}$ tolerance [34], and also supports that of our previous study, which demonstrated that SM593 had 
stronger aciduricity than SM18 in planktonic [24]. Biofilm was proposed to play a crucial role in acid tolerant of $S$. mutans [34]. In our previous study, we also found that SM593 exhibited a superior capacity to form more complex biofilm than SM18 and ATCC 25175 [35]. Therefore, we speculate that more complex structure of biofilm and greater ability of SM593 to form biofilm could partly explain a stronger capability of SM593 biofilm to tolerate low $\mathrm{pH}$ and grow compared with SM18 biofilm and ATCC 25175. Moreover, S. mutans genotypes from caries-free and caries-active individuals also differed in their ability to withstand acid challenge, and we extrapolate that the differences in caries susceptibility may be attributed to the colonization of specific strain, such as SM593. Whereas, it is worthwhile to note that there was no statistical difference in viability between SM18 and ATCC 25175. This may implicate that the virulence of standard strain ATCC 25175 was similar to that of SM18.

For each strain, the viability was highest in the middle biofilm layer while lowest in the inner layer. The viability varied from inner layer to outer layer because bacterial cells at different locations within a biofilm might not sense the same degree of $\mathrm{pH}$ stress simultaneously, and nutrient availability might be restricted when biofilm became thicker. This could be partly explained by the facts as reported in earlier reports [36-38]. Viable cells of the inner layer were least under different conditions, a plausible explanation of which probably lying in that relatively inadequate nutrition might slow their growth. Additionally, cells' metabolites might be detained in the inner layer, which possibly affected their growth as well. On the contrary, there were significantly more viable cells in the middle layer, where there was a more anaerobic condition and higher supply of nutrition, and inner and outer biofilm layers protected the cells in the middle biofilm layer against adverse stimulation from the environment stress. It was indicated that middle layer is likely the most suitable layer for bacteria to survive. The lower viability in the outer layer can be explained by the hypothesis that bacteria in this layer were directly exposed to adverse environmental stimulations which resulted in severe damage, although there was abundant nutrition for their growth. In addition, a similar viability distribution of the three layers in these strains of S. mutans also suggest that standard strain ATCC 25175 and clinical strains SM593 and 18 may have similar effect on regulating biofilm structure.

Nevertheless, the limitation of the present study focusing only on single-species biofilms of S. mutans should be noted. Multi-species biofilm formed by several cariogenic microorganisms, which is more close to natural dental plaque biofilms, need to be discussed in further study to complement the result of the current study.

\section{Materials and Methods}

\subsection{Bacterial Strains and Culture Conditions}

All experiments described herein were approved by the component local authorities. All procedures were done in agreement with National Institutes of Health guidelines. SM593 was isolated from caries-active adult who had multiple active carious lesions (the number of decayed and filled teeth $(D F T)=10$, no missing tooth, and three non-restored cavities). SM18 was isolated from caries-free adult (the number of decayed, missing, and filled teeth $(\mathrm{DMFT})=0$ ) [23]. S. mutans standard strain ATCC 25175, friendly provided by State Key Laboratory of Oral Diseases, Sichuan University (Chengdu, China), was applied as reference. Bacteria were cultured in tryptone-polypeptone-yeast extract (TPY) (Oxoid, Hampshire, England, UK) at $37^{\circ} \mathrm{C}$ in a controlled anaerobic environment $\left(80 \% \mathrm{~N}_{2}, 10 \% \mathrm{CO}_{2}\right.$, and $10 \% \mathrm{H}_{2}$ ). Pure cultures of each test strain were obtained and resuspended in fresh TPY with an optical density (OD) of 1.0 at $630 \mathrm{~nm}$ (approximately $1 \times 10^{8}$ cells $/ \mathrm{mL}$ ) for following experiments.

\subsection{Formation of Young and Mature Biofilms}

S. mutans biofilms were formed on sterile polystyrene plastic sheets $(1 \mathrm{~mm} \times 1 \mathrm{~mm}$, Dow Corning, Germany) which were immersed in Petri dishes containing $19 \mathrm{~mL}$ of TPY and $1 \mathrm{~mL}$ of resuspended bacterial suspension. The sheets were then incubated in an anaerobic chamber at $37^{\circ} \mathrm{C}$. The young and mature biofilms were formed on the plastic sheets after $3 \mathrm{~h}$ and $20 \mathrm{~h}$ incubation, respectively. 


\subsection{Acid-Adaptive Response of Young and Mature Biofilms}

After the formation of young and mature biofilms, the biofilm sheets were rinsed with phosphate-buffered saline (PBS; $\mathrm{pH}=7.0$ ) to remove non-adherent cells and then randomly divided into acid-adapted and non-adapted groups. The non-adapted group was immersed directly in TPY medium of $\mathrm{pH} 3.0$ for $30 \mathrm{~min}$, whereas the acid-adapted group was first immersed in TPY medium of $\mathrm{pH} 5.5$ for $3 \mathrm{~h}$ and then into the medium of $\mathrm{pH} 3.0$ for $30 \mathrm{~min}$.

\subsection{Acid Shock of Starved/Non-Starved Biofilms}

After $20 \mathrm{~h}$ of biofilm formation, all sheets were rinsed in $1 \mathrm{~mL}$ PBS $(\mathrm{pH}=7.0)$ to remove non-adherent cells, and then divided into non-starved and starved group randomly. The biofilms in the non-starved group were incubated in $20 \mathrm{~mL}$ TPY medium for $24 \mathrm{~h}$ and then immersed in TPY medium of $\mathrm{pH} 3.0$ for $30 \mathrm{~min}$. The biofilms of the starved group were immersed in PBS for $24 \mathrm{~h}$ and then into TPY medium of $\mathrm{pH} 3.0$ for $30 \mathrm{~min}$.

\subsection{Fluorescence Staining for Biofilms (Viability Staining)}

The Live/Dead BacLight Bacterial Viability Kit (L-7012, Molecular Probes, Eugene, OR, USA) contains SYTO-9 and propidium iodide (PI), which both stain nucleic acids. The kit was used to implement fluorescence staining for biofilms, following the manufacturer's instructions. Briefly, both SYTO-9 and PI diluted by distilled water at a ratio of 1.5:1000 and then thoroughly oscillated. Viable bacterial cells were stained with SYTO-9 (green), and bacterial cells with damaged membranes were stained with PI (red). The biofilms were removed from the culture media and washed with PBS to remove non-adherent cells. Each biofilm was immersed in $200 \mu \mathrm{L}$ of the corresponding stain, incubated for $15 \mathrm{~min}$ in the dark at room temperature, and then rinsed with $200 \mu \mathrm{L}$ of PBS. Next, $p$-phenylenediamine (5 mM) in PBS was added on top of the biofilm, which was then covered with a glass cover slip and viewed with CLSM.

\subsection{CLSM Observation of Biofilms and Image Analysis}

The stained biofilms were examined using a Zeiss LSM 510 CLSM (Carl Zeiss Microscopy, Germany). For each experiment, all parameters, including laser intensity, background level, contrast and zoom, were maintained at the same level. At $5 \mu \mathrm{m}$ intervals, a series of optical sections were acquired from the surface through the vertical axis of the specimen using a computer-controlled motor drive. Each biofilm was scanned at four randomly selected positions, and three series of optical sections were generated at each position. Image stacks were analyzed using the Java-based image analysis program Image J (Version 1.38, National Institutes of Health, Bethesda, MD, USA). Image J was used to count green (live cells) and red (dead cells) areas of the biofilms. The three-dimensional architecture of the biofilm was visualized with Zeiss LSM software. The percentage of live bacterial cells (viability) was calculated as a result of green areas/(red areas + green areas).

\subsection{Statistical Analysis}

The data were statistically analyzed using Statistical Package for Social Sciences (SPSS, version 20.0 software (Chicago, IL, USA). Results of the cell viability were reported as mean $\pm \mathrm{SD}$. One way analysis of variance (ANOVA) and t-test were used to compare means among three strains (SM593, SM18 and ATCC 25175) and between two different conditions (such as, young biofilm vs. mature biofilm, acid-adapted vs. non-adapted, starved vs. non-starved), respectively. $p<0.05$ was considered significantly different. Each experiment included six samples. Each assay was carried out in triplicate.

\section{Conclusions}

In conclusion, both ATR and starvation can enhance the aciduricity of biofilm formed by S. mutans regardless of strains. In addition, mature biofilm is more acid-resistant than young biofilm. 
Furthermore, SM593 from caries-active individuals exhibits a higher aciduricity than SM18 from caries-free individuals and standard strain ATCC 25175 in all cases, which may contribute to a better understanding of the differences in caries activity observed among S. mutans-infected individuals. The current study provides a potential and novel way to detect the cariogenic mechanism of S. mutans and to develop measures for caries prevention and management, especially for individuals with high risk of caries.

Acknowledgments: This work was supported by grants from the Natural Sciences Foundation of China (Grant Number: 30500564 and Grant Number: 81600861) and Joint Funds for the Innovation of Science and Technology, Fujian province (Grant Number: 2016Y9024).

Author Contributions: Shan Jiang performed the experiments, analysed the data, written and finalized the manuscript; Shuai Chen performed the experiments, written and finalized the manuscript; Chengfei Zhang designed the experiments and finalized the manuscript; Xingfu Zhao analysed the data; Xiaojing Huang conceived and designed the experiments, edited and finalized the manuscript; Zhiyu Cai analysed the data and finalized the manuscript.

Conflicts of Interest: The authors declared no conflict of interest.

$\begin{array}{ll}\text { Abbreviations } & \\ \text { ATCC } & \text { American Type Culture Collection } \\ \text { ANOVA } & \text { One way analysis of variance } \\ \text { ATR } & \text { Acid tolerance response } \\ \text { CLSM } & \text { Confocal laser scanning microscopy } \\ \text { DMFT } & \text { decayed, missing, and filled teeth } \\ \text { DFT } & \text { decayed and filled teeth } \\ \text { MS } & \text { Mutans Streptococcus } \\ \text { OD } & \text { Optical density } \\ \text { PBS } & \text { Phosphate-buffered saline } \\ \text { PCR } & \text { Polymerase chain reaction } \\ \text { PI } & \text { Propidium iodide } \\ \text { TPY } & \text { Tryptone-polypeptone-yeast extract } \\ \text { SD } & \text { Standard deviation } \\ \text { SPSS } & \text { Statistical Package for Social Science }\end{array}$

\section{References}

1. Featherstone, J.D. The continuum of dental caries-Evidence for a dynamic disease process. J. Dent. Res. 2004, 83, C39-C42. [CrossRef] [PubMed]

2. Featherstone, J.D. Dental caries: A dynamic disease process. Aust. Dent. J. 2008, 53, 286-291. [CrossRef] [PubMed]

3. Shemesh, M.; Tam, A.; Steinberg, D. Expression of biofilm-associated genes of Streptococcus mutans in response to glucose and sucrose. J. Med. Microbiol. 2007, 56, 1528-1535. [CrossRef] [PubMed]

4. Carlsson, J. Bacterial metabolism in dental biofilms. Adv. Dent. Res. 1997, 11, 75-80. [CrossRef] [PubMed]

5. Liu, S.; Tao, Y.; Yu, L.; Zhuang, P.; Zhi, Q.; Zhou, Y.; Lin, H. Analysis of Small RNAs in Streptococcus mutans under Acid Stress-A New Insight for Caries Research. Int. J. Mol. Sci. 2016, 17, 1529. [CrossRef] [PubMed]

6. Svensater, G.; Larsson, U.B.; Greif, E.C.; Cvitkovitch, D.G.; Hamilton, I.R. Acid tolerance response and survival by oral bacteria. Oral Microbiol. Immunol. 1997, 12, 266-273. [CrossRef] [PubMed]

7. Guo, L.; McLean, J.S.; Lux, R.; He, X.; Shi, W. The well-coordinated linkage between acidogenicity and aciduricity via insoluble glucans on the surface of Streptococcus mutans. Sci. Rep. 2015, 5, 18015. [CrossRef] [PubMed]

8. Landini, P.; Antoniani, D.; Burgess, J.G.; Nijland, R. Molecular mechanisms of compounds affecting bacterial biofilm formation and dispersal. Appl. Microbiol. Biotechnol. 2010, 86, 813-823. [CrossRef] [PubMed]

9. Fletcher, M. The physiological activity of bacteria attached to solid surfaces. Adv. Micro Physiol. 1991, 32, 53-85. 
10. Burne, R.A. Oral streptococci... products of their environment. J. Dent. Res. 1998, 77, 445-452. [CrossRef] [PubMed]

11. Welin, J.; Wilkins, J.C.; Beighton, D.; Wrzesinski, K.; Fey, S.J.; Mose-Larsen, P.; Hamilton, I.R.; Svensater, G. Effect of acid shock on protein expression by biofilm cells of Streptococcus mutans. FEMS Microbiol. Lett. 2003, 227, 287-293. [CrossRef]

12. Arnold, K.W.; Kaspar, C.W. Starvation-And stationary-phase-induced acid tolerance in Escherichia coli O157:H7. Appl. Environ. Microbiol. 1995, 61, 2037-2039. [PubMed]

13. Zhu, M.; Takenaka, S.; Sato, M.; Hoshino, E. Influence of starvation and biofilm formation on acid resistance of Streptococcus mutans. Oral Microbiol. Immunol. 2001, 16, 24-27. [CrossRef] [PubMed]

14. Washio, J.; Takahashi, N. Metabolomic Studies of Oral Biofilm, Oral Cancer, and Beyond. Int. J. Mol. Sci. 2016, 17, 870. [CrossRef] [PubMed]

15. Lembo, F.L.; Longo, P.L.; Ota-Tsuzuki, C.; Rodrigues, C.R.; Mayer, M.P. Genotypic and phenotypic analysis of Streptococcus mutans from different oral cavity sites of caries-free and caries-active children. Oral Microbiol. Immunol. 2007, 22, 313-319. [CrossRef] [PubMed]

16. Guo, L.H.; Shi, J.N.; Zhang, Y.; Liu, X.D.; Duan, J.; Wei, S. Identification of genetic differences between two clinical isolates of Streptococcus mutans by suppression subtractive hybridization. Oral Microbiol. Immunol. 2006, 21, 372-380. [CrossRef] [PubMed]

17. Guo, L.H.; Wang, H.L.; Liu, X.D.; Duan, J. Identification of protein differences between two clinical isolates of Streptococcus mutans by proteomic analysis. Oral Microbiol. Immunol. 2008, 23, 105-111. [CrossRef] [PubMed]

18. Loesche, W.J. Role of Streptococcus mutans in human dental decay. Microbiol. Rev. 1986, 50, 353-380. [PubMed]

19. Van Houte, J.; Jordan, H.V.; Laraway, R.; Kent, R.; Soparkar, P.M.; DePaola, P.F. Association of the microbial flora of dental plaque and saliva with human root-surface caries. J. Dent. Res. 1990, 69, 1463-1468. [CrossRef] [PubMed]

20. Giacaman, R.A.; Araneda, E.; Padilla, C. Association between biofilm-forming isolates of mutans streptococci and caries experience in adults. Arch. Oral Biol. 2010, 55, 550-554. [CrossRef] [PubMed]

21. Huang, X.; Liu, T.; Chen, Z.; Zhan, L.; Yang, J. Evaluation of cariogenic potential of Streptococcus mutans isolated from caries-free and -active persons: Adherence properties to saliva-coated hydroxyapatite. Hua Xi Kou Qiang Yi Xue Za Zhi 2000, 18, 416-418. [PubMed]

22. Huang, X.; Liu, T.; Yang, J.; Chen, Z.; Liu, J. Evaluation of cariogenic potential of Streptococcus mutans isolated from caries-free and -active persons: Abilities to synthesize water-soluble and-Insoluble glucans. Hua Xi Kou Qiang Yi Xue Za Zhi 2000, 18, 419-421. [PubMed]

23. Huang, X.; Liu, T.; Chen, G. [Typing of Streptococcus mutans (serotype C) by arbitrarily primed polymerase chain reaction]. Zhonghua Kou Qiang Yi Xue Za Zhi 2001, 36, 281-284. [PubMed]

24. Huang, X.J.; Liu, T.J.; Cai, Z.Y.; Chen, Z.; Yang, J.B.; Liu, J.G. [Evaluation of the in vitro cariogenic potential of Streptococcus mutans (serotype C) strains isolated from caries-free and -active people: The ability of acidogenicity]. Sichuan Da Xue Xue Bao Yi Xue Ban 2004, 35, 520-521. [PubMed]

25. Belli, W.A.; Marquis, R.E. Adaptation of Streptococcus mutans and Enterococcus hirae to acid stress in continuous culture. Appl. Environ. Microbiol. 1991, 57, 1134-1138. [PubMed]

26. Hamilton, I.R.; Buckley, N.D. Adaptation by Streptococcus mutans to acid tolerance. Oral Microbiol. Immunol. 1991, 6, 65-71. [CrossRef] [PubMed]

27. Motegi, M.; Takagi, Y.; Yonezawa, H.; Hanada, N.; Terajima, J.; Watanabe, H.; Senpuku, H. Assessment of genes associated with Streptococcus mutans biofilm morphology. Appl. Environ. Microbiol. 2006, 72, 6277-6287. [CrossRef] [PubMed]

28. Svensater, G.; Sjogreen, B.; Hamilton, I.R. Multiple stress responses in Streptococcus mutans and the induction of general and stress-specific proteins. Microbiology 2000, 146, 107-117. [CrossRef] [PubMed]

29. Wilkins, J.C.; Homer, K.A.; Beighton, D. Analysis of Streptococcus mutans proteins modulated by culture under acidic conditions. Appl. Environ. Microbiol. 2002, 68, 2382-2390. [CrossRef] [PubMed]

30. Shen, Y.; Stojicic, S.; Haapasalo, M. Antimicrobial efficacy of chlorhexidine against bacteria in biofilms at different stages of development. J. Endod. 2011, 37, 657-661. [CrossRef] [PubMed]

31. Shen, Y.; Zhao, J.; de la Fuente-Nunez, C.; Wang, Z.; Hancock, R.E.; Roberts, C.R.; Ma, J.; Li, J.; Haapasalo, M.; Wang, Q. Experimental and Theoretical Investigation of Multispecies Oral Biofilm Resistance to Chlorhexidine Treatment. Sci. Rep. 2016, 6, 27537. [CrossRef] [PubMed] 
32. Costerton, J.W.; Cheng, K.J.; Geesey, G.G.; Ladd, T.I.; Nickel, J.C.; Dasgupta, M.; Marrie, T.J. Bacterial biofilms in nature and disease. Annu. Rev. Microbiol. 1987, 41, 435-464. [CrossRef] [PubMed]

33. Sonia Goodacre, C.C.; Slattery, C. Cambridge VCE Health and Human Development Units 1 and 2 App, 2nd ed.; Cambridge University Press: Cambridge, UK, 2014.

34. Welin-Neilands, J.; Svensater, G. Acid tolerance of biofilm cells of Streptococcus mutans. Appl. Environ. Microbiol. 2007, 73, 5633-5638. [CrossRef] [PubMed]

35. Jiang, S.; Huang, X.; Zhang, C.; Cai, Z.; Zou, T. Morphological and proteomic analyses of the biofilms generated by Streptococcus mutans isolated from caries-active and caries-free adults. J. Dent. Sci. 2015, 10, 206-215. [CrossRef]

36. Parsek, M.R.; Greenberg, E.P. Acyl-homoserine lactone quorum sensing in gram-negative bacteria: A signaling mechanism involved in associations with higher organisms. Proc. Natl. Acad. Sci. USA 2000, 97, 8789-8793. [CrossRef] [PubMed]

37. Li, Y.H.; Lau, P.C.; Tang, N.; Svensater, G.; Ellen, R.P.; Cvitkovitch, D.G. Novel two-component regulatory system involved in biofilm formation and acid resistance in Streptococcus mutans. J. Bacteriol. 2002, 184, 6333-6342. [CrossRef] [PubMed]

38. Ahmed, N.A.; Petersen, F.C.; Scheie, A.A. Biofilm formation and autoinducer-2 signaling in Streptococcus intermedius: Role of thermal and pH factors. Oral Microbiol. Immunol. 2008, 23, 492-497. [CrossRef] [PubMed]

(C) 2017 by the authors. Licensee MDPI, Basel, Switzerland. This article is an open access article distributed under the terms and conditions of the Creative Commons Attribution (CC BY) license (http:/ / creativecommons.org/licenses/by/4.0/). 